\title{
Low-cost Low-power Small-form-factor Node for Health Monitoring Wireless Multi-Hop Personal Area Networks with Dramatically Increased Range
}

\author{
V. Lakafosis*, N. Chaisilwattana, C. Kruesi, L. Yang, D. Staiculescu, M.M. \\ Tentzeris \\ Georgia Institute of Technology, Georgia Electronic Design Center, Atlanta, GA, 30308, \\ U.S.A. \\ e-mail: vasilis@ece.gatech.edu
}

\section{Introduction}

The medical device field is an emerging technology that has captured the interest of many engineers. The increased life expectancy created the need for reliable real time vital signal monitoring from the comfort of the home, to be transmitted to an Internet database for continuous access of medical personnel. Standard techniques for measurement of ECG signals or body temperature use bulky wires in order to measure the very small currents inside the body [1]. To allow for the flexibility in patient mobility, health monitoring Personal Area Networks have emerged, where autonomous nodes sense biological signals and transmit them through wireless ad hoc multi-hopping networks back to a base station for processing and accurate ECG reporting. In this paper, we present the system architecture of a small-sized wireless sensor node for simultaneous ECG and temperature monitoring, manage to overlay this information through wireless multi-hop routes consisting of other such nodes-patients to a sink and investigate the radiation properties of antennas close to the human body, which will later be integrated with the sensor node. Currently, there is difficulty in the communication between sensors over the body because the omni-directional monopole antenna commonly used is not efficient and has losses due to the proximity to the human body. The proposed patch antenna operates at $915 \mathrm{MHz}$ and fits comfortably on the human body.

\section{System Architecture}

The proposed Wireless Personal Area Network has been designed to serve as the backbone of the health monitoring system and is composed of low-power, low-cost, miniaturized wireless sensor motes that, besides processing and communication capabilities, possess two human vital signs sensors.

The required communication and processing functionality is offered by the commercially available Xbow's MICA wireless sensor node; lower board shown in Fig. 1. This board's size dimensions are $1.5 \times 2$ inch and dictated the cross-section size of whole patient-centered wireless node. Its TI CC1000 transceiver was configured to operate in $915 \mathrm{MHz}$ with low RF transmit power offering single-hop range of a few tenths of meters and bandwidth on the order of a few hundred kbps. Two of the node's onboard 8-bit A-to-D converters were used for the conversion of the output voltages of the ECG and body temperature sensors to binary digits so that they can be transmitted, as mentioned later, to the base station. For its powering, the $3.7 \mathrm{~V}_{\mathrm{DC}}$ voltage of a slim 1400 $\mathrm{mAh}$ battery had to be converted to the standard $3 \mathrm{~V}$ with a voltage regulator.

On top of the aforementioned communication and processing board is the sensing board; upper board shown in Fig. 1. First of all, this printed circuit board houses a custom 3-lead ECG sensor an output of which is shown in Fig. 2. This very reliable 
electrocardiogram of the electrical activity of the heart over time corresponds to an $\mathbf{8 0}$ beats per minute output of the BC Biomedical's MaxiSim 2000 patient simulator. The current draw is low and the op amps are reliably powered with 4 AAA batteries. Additionally, the patient's body temperature is measured with the medical grade YSI400 temperature probe, the output of which is variable resistance and, thus, has to be converted to variable voltage within a desired domain, suitable for the ADC to "read". It is obvious that this board is an early prototype one and can enormously be miniaturized by using surface mount components.

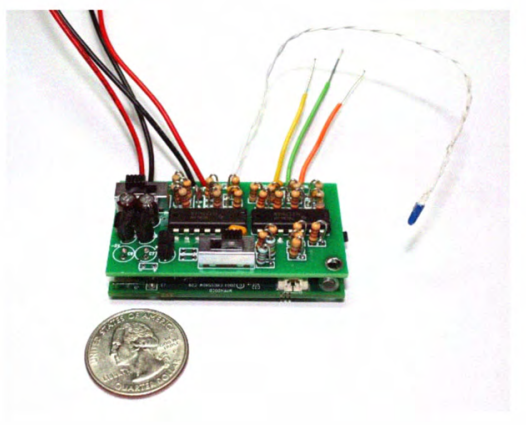

Fig. 1 Sensor node implementation

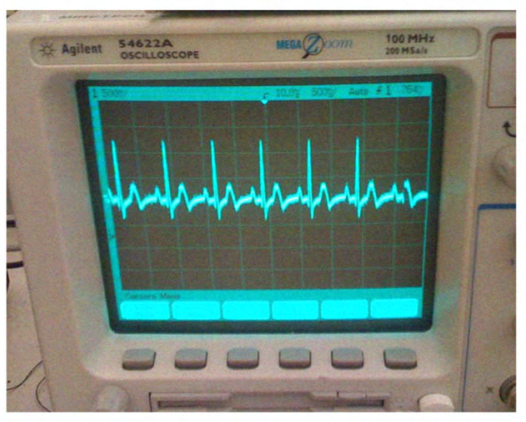

Fig. 2 Transmitted ECG signal captured on oscilloscope

The patient-centered nodes comprising an "ad-hoc" wireless sensor network are interconnected with wireless links and are capable of hopping radio messages to a base station where they are passed to a data base, as depicted in fig. 3. The networking service required above the physical layer is offered by XMesh, a full featured multi-hop, ad-hoc, mesh networking protocol developed by Crossbow [3], which meets the requirements posed by the challenging medical monitoring environment.

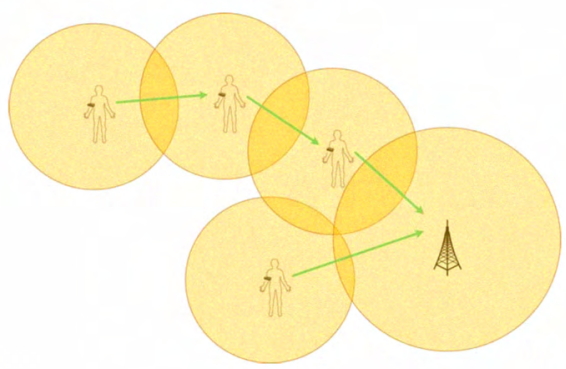

Fig. 3 Health monitoring PAN

First of all, longer-term operation of these compact battery-powered devices is achieved by minimizing the power consumption of the radio transmission. Instead of transmitting high RF strength signals over long single-hop wireless links, it is more power efficient to relay packets a number of times over lower strength shorter links, as discussed in [2]. At the same time, as long as nodes are located within direct radio range of others, hopping effectively extends radio communication over higher ranges overcoming non-line-of-sight and path loss effects. Reliability, which is of vital importance, is also boosted by the fact that guaranteed delivery is supported with end-toend acknowledgements and that the topology is both self-organizing and self-healing. Regarding the latter feature in particular, not only does the topology have the ability to sustain sensor network functionalities without any interruption due to sensor node failures but also because of the bi-directional communication the base station can adjust 
certain operating parameters of the sensors, in response to health information received including radio traffic, battery voltage and parent's node radio signal strength indicator.

As is the case with any wireless network, the purpose of the cost metric is to minimize the total cost it takes for any mote to transmit to the base station. It is important to note that, instead of hop count which is the traditional distance vector routing cost metric, XMesh uses the Minimum Transmission cost metric which minimizes the total number of transmissions in delivering a packet over multiple hops to a destination.

\section{Antenna design}

Recently, there have been many publications incorporating fabric with wearable antennas because of their enhanced comfort. Many of these also use electrically conductive fabric for the patch and ground plane. Liquid Crystal Polymer (LCP), a material that has been studied and used quite effectively in recent years, is also considered. A disadvantage of this material is that it is typically used for high frequency or planar dipole antennas, and thus is only manufactured as a very thin substrate (maximum $0.1 \mathrm{~mm}$ ). This would mean that, in order to effectively fabricate the antenna, layers of LCP would need to be bonded together to form a substrate. Although feasible, it may not be practical for a solution in the disposable medical devices industry due to a higher manufacturing cost. The material that was considered for this experiment is Rogers Corporation TMM 10i material. It is a lower cost material that had advantageous dielectric properties, allowing for the miniaturization of the antenna. Also, it can be fabricated using the same process as conventional PCBs, which reduces manufacturing time and cost. The dielectric properties of these materials are: $\varepsilon_{\mathrm{r}}=9.8$ and $\tan _{\delta}=0.002$.

In designing the circular patch, we want to excite the lowest order mode, the $\mathrm{TM}_{11}$ mode. The substrate thickness for the TMM $10 \mathrm{i}$ material is $2.54 \mathrm{~mm}$. The simulation was performed in Ansoft's HFSS simulation software with a ground plane equal to 1.75 times the radius in order to minimize fringing fields without making the overall size of the antenna too large. The computed [4] and optimized values for the radius of the circular patch antennas for the different substrates are given in Table 1 . The feed point was about $1 / 3$ of the radius away from the center [4].

\begin{tabular}{|c|c|c|c|}
\hline Material & Computed rad $(\mathrm{mm})$ & Optimized rad $(\mathrm{mm})$ & Antenna diam $(\mathrm{cm})$ \\
\hline Polyester & $67.4 \mathrm{~mm}$ & $69.2 \mathrm{~mm}$ & $24.22 \mathrm{~cm}$ \\
\hline LCP & $54.1 \mathrm{~mm}$ & $55.1 \mathrm{~mm}$ & $19.29 \mathrm{~cm}$ \\
\hline TMM 10i & $30.3 \mathrm{~mm}$ & $30.8 \mathrm{~mm}$ & $10.78 \mathrm{~cm}$ \\
\hline
\end{tabular}

Table 1. Antenna size for various materials

The antenna fabricated on the Rogers TMM 10i substrate seems the best solution for our application due to the smaller size. Although the efficiency is limited due to the large dielectric constant, we are not as limited by this factor, since the antennas will be used within a confined space and do not need to be able to transmit further than a few meters.

To better incorporate the Rogers TMM 10i antenna into a medical environment, we can coat the outside of the antenna with Silicone, a material used in implantable devices and widely disseminated in hospitals. Pure Silicone has been analyzed as having an $\varepsilon_{\mathrm{r}}$ of 2.65 and $\tan _{\delta}$ of 0.001 . Dielectric layering will cause a frequency shift in the antenna to a lower frequency, and thus allow for further miniaturization of the antenna [5]. After adjusting the size of the antenna to have a resonance at $915 \mathrm{MHz}$, the dimensions of the antenna are a patch radius of $30.4 \mathrm{~mm}$, and a total diameter of 10.64 
$\mathrm{cm}$. The gain in the broad-side direction is $0.55 \mathrm{~dB}$. The geometry and magnitude of $\mathrm{S} 11$ of the antenna are shown in fig. 4 and 5, respectively.

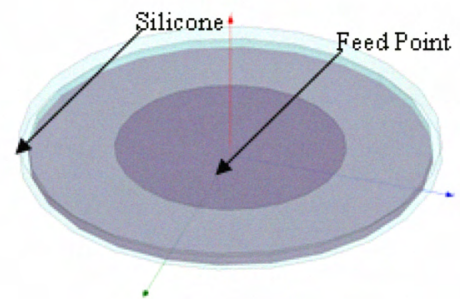

Fig. 4 Circular Patch covered with Silicone

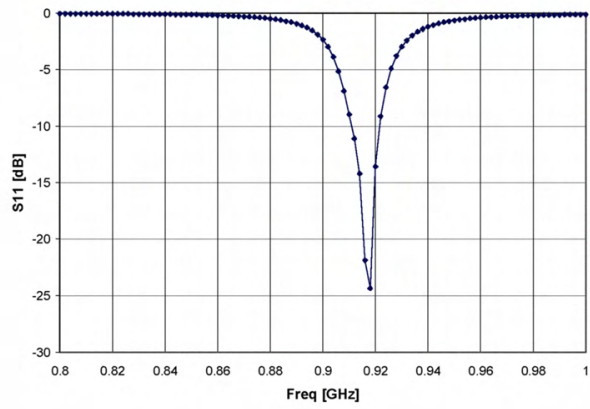

Fig. $5 \mathrm{~S}_{11}$ of the Silicone covered antenna

Using the FEKO homogenous human body phantom, the radiation pattern of the antenna placed on the body can be simulated. These results are shown in Fig. 6. Care must be taken when integrating the antenna with the human body phantom. A FEM current source must be used in place of the SMA connection, which was used for the HFSS calculations, and the antenna structure must be volume meshed, as shown in Fig. 6. The radiated power is directed away from the human body, which limits the specific absorption rate into the body.
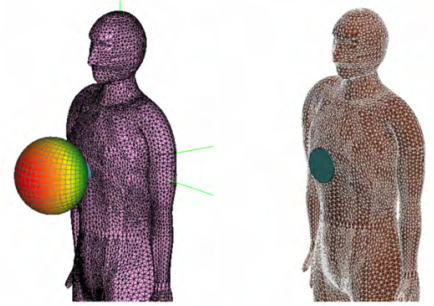

Fig. 6 Radiation pattern of antenna on human phantom using FEKO

\section{Conclusions}

The individual components comprising a low-cost, low-power, small-form-factor wireless sensor node for simultaneous ECG and temperature monitoring over wireless personal area networks of dramatically increased range, have been successfully designed, tested and presented. A circular patch antenna has been created that can be integrated, the substrate material of which has been chosen in order to optimize size and reduce radiation lost from interactions with the body. In the future, our work will focus on adding extra reliability measures related to data traffic issues, introducing security layers in the WPAN and optimizing the design of the patch antenna for further miniaturization.

\section{References}

[1] J. C. Lin, "Biomedical Applications of Electromagnetic Engineering", Engineering Electromagnetics Applications, R. Bansal, Ed. Boca Raton: CRC Press, 2006, pp. 211-233.

[2] V. Lakafosis, M.M. Tentzeris, "Implementation of multi-hop routing protocols for the dramatic range enhancement of wireless sensor networks", IEEE Antennas and Propagation Society International Symposium, July 2008.

[3] Crossbow Technologies, XMesh User's Manual, Revision D, April 2007.

[4] T. Milligan, "Modern Antenna Design", $2^{\text {nd }}$ Ed., Hoboken, NJ: IEEE Press, 2005, pp. 313-315

[5] I. Bahl, P. Bhartia, S. Stuchly, "Design of Microstrip Antennas Covered with a Dielectric Layer", IEEE Transactions on Antennas and Propagation, vol. 30, no. 2, pp. 314-318, March 1982 